\title{
ESTUDO SEMI-EMPÍRICO DE ÁCIDOS HIDROXÂMICOS: ÁCIDO FORMOIDROXÂMICO E DERIVADOS DO ALELOQUÍMICO DIMBOA
}

\section{Carlos Mauricio R. Sant'Anna*}

Departamento de Química, ICE, Universidade Federal Rural do Rio de Janeiro, 23851-970 Seropédica - RJ

Departamento de Fármacos, Universidade Federal do Rio de Janeiro, Cidade Universitária, CCS, 21949-910 Rio de Janeiro - RJ

Vivian Passos de Souza

Departamento de Química, ICE, Universidade Federal Rural do Rio de Janeiro, 23851-970 Seropédica - RJ

Recebido em 22/3/00; aceito em 5/2/01

\begin{abstract}
A SEMIEMPIRICAL STUDY ON HYDROXAMIC ACIDS: FORMOHYDROXAMIC ACID AND DERIVATIVES OF THE ALLELOCHEMICAL DIMBOA. Open chain hydroxamic acid (Hx) can exist as $Z$ and $E$ diastereomers of two tautomers, hydroxamic acid and hydroximic acid. The conformational stability of the formohydroxamic acid isomers evaluated by PM3 compared better to ab initio results from the literature than AM1 results. Structural data of the cyclic Hx 2,4-dihydroxy-7-metoxy-2H-1,4benzoxazin-3(4H)-one (DIMBOA) obtained by both semiempirical methods compared well to ab initio results. $\mathrm{p} K_{\mathrm{a}}$ data from the literature for derivatives of the aldolic isomer of DIMBOA were compared to the stability of the anions resulting from the loss of protons of their phenol and hydroxamic acid groups, determined as the difference in heat of formation between anionic and neutral forms, calculated by AM1 and PM3 methods. Good correlations between theoretical and experimental data were obtained for both semiempirical methods.
\end{abstract}

Keywords: semiempirical; hydroxamic acids; acidity.

\section{INTRODUÇÃO}

Todos os anos a produção mundial de grãos sofre grandes perdas, tanto em quantidade quanto em qualidade, devido à infestação por pragas, principalmente insetos. Além do custo elevado, o uso em larga escala de inseticidas sintéticos para o controle dos insetos tem resultado na perda de eficiência devido ao desenvolvimento de resistência pelos insetos e em efeitos danosos sobre o meio ambiente. A investigação dos mecanismos naturais de defesa das plantas contra organismos agressores é uma possível fonte de alternativas para o desenvolvimento de novas classes de inseticidas. As plantas produzem metabólitos secundários, chamados substâncias alelopáticas ou aleloquímicos, que atuam na sua defesa contra herbívoros, patógenos ou mesmo contra plantas competidoras ${ }^{1}$.

Estudos têm demonstrado que ácidos hidroxâmicos $(\mathrm{Hx})$ cíclicos do tipo 4-hidróxi-1,4-benzoxazin-3-onas (benzoxazinóides), presentes em cereais na forma de 2 - $\beta$ - $O$-D-glicosídeos, atuam sobre insetos, fungos e bactérias ${ }^{2-5}$. Os glicosídeos são biologicamente inativos, mas a ruptura das células vegetais libera $\beta$-glicosidases que degradam os glicosídeos enzimaticamente, liberando as agliconas tóxicas ${ }^{6}$.

O Hx predominante no trigo e no milho é a 2,4-diidroxi-7metoxi-1,4-benzoxazin-3-ona (DIMBOA, 1, Figura 1), isolada sob a forma do glicosídeo ( $2 R$ )-2- $\beta$-glicopiranosiloxi-4-hidroxi7-metoxi-2H-1,4- benzoxazin-3-ona ${ }^{6}$. Em solução aquosa, 1 está em equilíbrio com a forma aldólica 2 (Figura 1$)^{7}$. A participação desse Hx na defesa do trigo contra insetos é sugerida pelo aumento de seus níveis na planta após infestação por afídios ${ }^{3,5}$ e pelo seu efeito anti-alimentar em insetos alimentados com dietas artificiais contendo $\mathbf{1}^{8}$. Existem várias indicações quanto aos possíveis sítios bioquímicos de ação dos ácidos hidroxâmicos. Foi demonstrado que 1 inibe enzimas envolvidas na síntese de $\mathrm{ATP}^{9}$, a acilcolinaesterase (acilcolina hidrolase $)^{10}$, a enzima destoxificante UDP-glicose transferase ${ }^{11}$

*e-mail: santanna@ufrrj.br e as proteinases papaina ${ }^{12}$ e $\alpha$-quimotripsina ${ }^{13}$. Apesar das duas últimas não serem enzimas de insetos, os resultados sugerem que enzimas digestivas de insetos podem estar entre os sítios de ação dos Hx.<smiles>C=C=CC</smiles>

Figura 1. 2,4-diidroxi-7-metoxi-1,4-benzoxazin-3-ona (DIMBOA) (1) e sua forma aldólica (2).

Os métodos semi-empíricos têm características interessantes para o estudo de mecanismos enzimáticos. Diferente dos métodos de campo empírico, a metodologia semi-empírica pode avaliar a formação e a quebra de ligações covalentes, por considerar explicitamente os elétrons através da resolução da equação de Schrödinger ${ }^{14}$. Em comparação com os métodos ab initio, simplificações introduzidas nos métodos semi-empíricos tornam viável o cálculo em computadores de baixo custo de sistemas moleculares com várias centenas de orbitais ${ }^{15}$, que podem abranger o substrato e os aminoácidos que compõe o sítio ativo e suas vizinhanças imediatas. Estas simplificações incluem a consideração apenas dos elétrons de valência, a restrição do conjunto de bases à representação mínima e a negligência, em graus variáveis, do recobrimento dos orbitais atômicos localizados em átomos diferentes ${ }^{14,15}$. Nos métodos desenvolvidos por Dewar e colaboradores, parâmetros empíricos foram introduzidos na busca da melhor correlação com dados experimentais, que incluem, por exemplo, geometrias de equilíbrio, calores de formação, potenciais de ionização e momentos de dipolo $^{15}$. Resultados satisfatórios foram obtidos para várias propriedades, mas deve-se ter em mente que os resultados são melhores quanto mais relacionados são os dados experimentais com aqueles que se deseja calcular ${ }^{15}$. 
Neste sentido, o objetivo do presente trabalho é avaliar o desempenho dos métodos semi-empíricos na modelagem de moléculas que contêm o grupamento ácido hidroxâmico em comparação com cálculos ab initio e dados experimentais da literatura, visando fornecer subsídios para futuros estudos da interação de $\mathrm{Hx}$ com modelos de sistemas enzimáticos de insetos com a metodologia semi-empírica. Estudos com campos empíricos foram feitos para sistemas enzimáticos inibidos por inibidores do tipo $\mathrm{Hx}$, mas os resultados limitam-se às etapas iniciais de interação ${ }^{16}$.

\section{RESULTADOS}

\section{Avaliação dos métodos semi-empíricos para a produção de dados estruturais}

Inicialmente, foi avaliado o desempenho dos métodos semiempíricos $\mathrm{AM} 1^{17}$ e $\mathrm{PM} 3^{18}$ na modelagem de estruturas simples contendo a função ácido hidroxâmico. Os Hx de cadeia aberta podem existir como diastereoisômeros Z/E das formas tautoméricas ácido hidroxâmico e ácido hidroxímico ${ }^{19}$. A estabilidade relativa das estruturas 3a a 3d (Figura 2) foi avaliada com os métodos semi-empíricos para o ácido formoidroxâmico. Os ângulos diedros em destaque foram avaliados com incrementos de $30^{\circ}$ entre $-180^{\circ}$ e $180^{\circ}$.

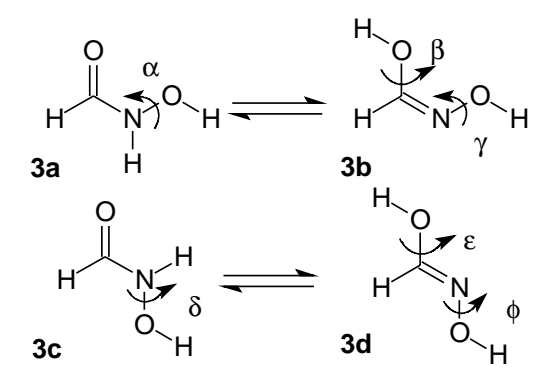

Figura 2. 3a, ácido formoidroxâmico, isômero $Z$; 3 b, ácido formoidroxímico, isômero $Z ; 3 c$, ácido formoidroxâmico, isômero $E ; 3 d$, ácido hidroxímico, isômero $E$.

A estrutura de menor energia pelo método PM3 é a conformação anti-clinal do ângulo $\delta(\mathrm{H}-\mathrm{O}-\mathrm{N}-\mathrm{C})$ do estereoisômero $E$ da forma ácido hidroxâmico (3c $a c$ ). Pelo método AM1, a estrutura mais estável é o estereoisômero $Z$ da forma aldólica ácido hidroxímico, nas conformações anti-periplanar para o ângulo $\gamma(\mathrm{H}-\mathrm{O}-\mathrm{N}-\mathrm{C})$ e sin-periplanar para o ângulo $\beta$ (H-O-CN) (3b $a p, s p)$. De forma geral, o desempenho do método PM3 é superior ao do método AM1: com exceção da estabilidade relativa das estruturas $\mathbf{3 b} a p, s p$ e $\mathbf{3 d} a p, s p$, a ordem de estabilidade prevista pelo método PM3 está de acordo com resultados da literatura ${ }^{19}$ obtidos pela otimização da estrutura com o método ab initio MP2/6-31G(d), seguida do cálculo de energia de ponto único com um conjunto de bases maior, 6-311G(d,p) (Tabela 1).

A Tabela 2 compara os dados dos métodos semi-empírico PM3 e ab initio MP2/6-311G(d,p)//MP2/6-31G (d) para as distâncias de ligação, ângulos de ligação e ângulos diedros da estrutura mais estável encontrada ${ }^{19}$. Há, de modo geral, uma boa semelhança entre os valores calculados pelos dois métodos; as maiores diferenças são observadas para a distância N$\mathrm{H}(9,80 \%)$, para o ângulo $\mathrm{H}-\mathrm{C}-\mathrm{N}(5,93 \%)$ e para o ângulo diedro H-N-C-O $(17,89 \%)$.

A Tabela 3 compara os dados de distâncias e ângulos de ligação para o Hx cíclico 1, obtidos pelo método ab initio $\mathrm{HF} /$ 6-31G* e pelos métodos semi-empíricos AM1 e PM3. Ambos os métodos semi-empíricos reproduzem de forma razoável os resultados do cálculo $a b$ initio. As maiores diferenças para as
Tabela 1. Estabilidade relativa calculada do ácido formoidroxâmico ${ }^{\mathrm{a}}$.

\begin{tabular}{cccc}
\hline Isômero & AM1 & PM3 & MP2/6-311G(d,p) \\
\hline 3c $a c$ & $-48,16^{\mathrm{b}}$ & $-45,85^{\mathrm{b}}$ & $-1,534.10^{5} \mathrm{c}$ \\
3a $s c$ & 0,85 & 1,33 & 0,31 \\
3a $a c$ & 1,87 & 2,60 & 1,29 \\
3b $a p, s p$ & $-2,84$ & 3,47 & 1,31 \\
3d $a p, s p$ & 1,34 & 2,41 & 5,10 \\
3b $a p, a p$ & 2,35 & 6,06 & 8,54 \\
3d $a p, a p$ & 7,51 & 6,16 & 9,69 \\
3b sp,ap & 2,59 & 10,31 & 10,02
\end{tabular}

${ }^{a}$ Energias e entalpias para todas as estruturas são relativas à estrutura $3 c$ ac, cujos valores de energia total e $\Delta \mathrm{H}_{\mathrm{f}}$ são apresentados; ${ }^{\mathrm{b}} \Delta \mathrm{H}_{\mathrm{f}}$, em $\mathrm{kcal} / \mathrm{mol}$; valores relativos em $\mathrm{kcal} / \mathrm{mol}$; ${ }^{\mathrm{c}}$ Energia, em $\mathrm{kcal} / \mathrm{mol}$; valores relativos em $\mathrm{kcal} / \mathrm{mol}^{22}$.

Tabela 2. Comparação dos parâmetros estruturais da estrutura, 3c ac, encontrada pelos métodos MP2/6-31G (d), PM3 e AM1

\begin{tabular}{cccccc}
\hline Parâmetro $^{\text {a,b }}$ & $\begin{array}{c}\text { MP2/6- } \\
\text { 31G }(d)^{22}\end{array}$ & AM1 & Diferença $^{c}$ & PM3 & Diferença $^{c}$ \\
\hline C-O & 1,221 & 1,236 & $-0,015$ & 1,213 & 0,008 \\
C-N & 1,388 & 1,404 & $-0,016$ & 1,429 & $-0,041$ \\
N-O & 1,420 & 1,325 & 0,095 & 1,437 & $-0,017$ \\
C-H & 1,102 & 1,109 & $-0,007$ & 1,101 & 0,001 \\
N-H & 1,102 & 1,012 & 0,090 & 0,994 & $-0,108$ \\
Média & - & - & 0,045 & - & 0,035 \\
O-C-N & 122,96 & 120,29 & 2,67 & 116,75 & 6,21 \\
C-N-O & 113,36 & 116,36 & $-3,00$ & 114,17 & $-0,81$ \\
H-C-N & 111,89 & 114,79 & $-2,90$ & 118,52 & $-6,63$ \\
H-N-O & 110,20 & 112,71 & $-2,51$ & 110,42 & $-0,22$ \\
H-O-N & 103,06 & 105,73 & $-2,67$ & 101,25 & 1,81 \\
Média & - & - & 2,75 & - & 3,14 \\
O-C-N-O & 156,80 & 159,3 & $-2,50$ & 158,02 & $-1,22$ \\
H-C-N-O & $-26,96$ & $-25,49$ & $-1,47$ & $-26,16$ & $-0,80$ \\
H-N-C-O & 29,55 & 21,40 & 8,15 & 24,56 & 4,99 \\
H-O-N-C & 119,27 & 120,10 & $-0,83$ & 118,52 & 0,75 \\
Média & - & - & 3,31 & - & 1,94 \\
\hline
\end{tabular}

${ }^{\mathrm{a}}$ Distância de ligações em $\AA$, ângulos e diedros em graus; ${ }^{\mathrm{b}} \mathrm{As}$ médias referem-se às diferenças, em módulo, para o conjunto de cada tipo de parâmetro (distância de ligação, ângulo de ligação e ângulo diedro); 'Parâmetro(ab initio) - Parâmetro(semi-empírico)

distâncias das ligações são observadas em C3-N4, 0,0884 ̊ $(6,53 \%)$ e $0,0811 \AA(5,99 \%)$ mais longas de acordo com os métodos PM3 e AM1, respectivamente, e N4-O13, 0,0888 $(6,49 \%)$ mais longa pelo método PM3. O ângulo O1-C2-O11 é $7,70^{\circ}(7,00 \%)$ maior de acordo com o método AM1 e $11,34^{0}$ $(10,31 \%)$ maior pelo método PM3 e o ângulo C3-N4-C10 é $6,97^{0}(5,72 \%)$ maior de acordo com o método AM1 e 5,32 $(4,37 \%)$ maior pelo método PM3.

Os dados de carga obtidas por ajuste ao potencial eletrostático são apresentados na Tabela 4. Diferenças consideráveis são observadas entre as cargas calculadas por ambos os métodos semi-empíricos e o método $a b$ initio, mas o método PM3 produz, mesmo para os heteroátomos, resultados mais próximos aos obtidos com o método $\mathrm{HF} / 6-31 \mathrm{G}^{*}$.

\section{Estudo dos efeitos eletrônicos sobre $\mathrm{pK}_{\mathrm{a}}$ de $\mathrm{Hx}$ pelos métodos semi-empíricos}

Uma segunda avaliação do desempenho dos método semiempíricos foi feita por comparação com dados experimentais ${ }^{20}$ de acidez dos grupos ácido hidroxâmico $\left(\mathrm{p} K_{\mathrm{a} 1}\right)$ e fenol $\left(\mathrm{p} K_{\mathrm{a} 2}\right)$ de análogos de 2 com diferentes substituintes em C7. Os valores de $\mathrm{p} K_{\mathrm{a}}$ se relacionam com o $\Delta \mathrm{G}_{\mathrm{r}}$ das reações de desprotonação de acordo com a conhecida relação: 
Tabela 3. Comparação das estruturas obtidas pelos métodos HF/6-31G*, AM1 e PM3.

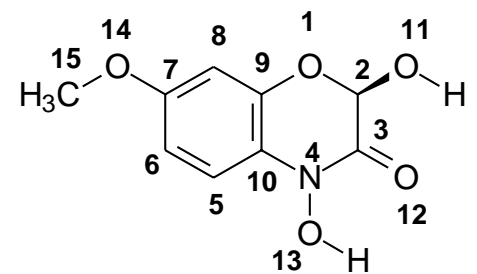

\begin{tabular}{|c|c|c|c|c|c|}
\hline Parâmetro ${ }^{\mathrm{a}, \mathrm{b}}$ & $\mathrm{HF} / 6-31 \mathrm{G}^{*}$ & AM1 & Diferença $\mathrm{a}^{\mathrm{c}}$ & PM3 & Diferenç $\mathrm{a}^{\mathrm{c}}$ \\
\hline $\mathrm{O} 1-\mathrm{C} 2$ & 1,408 & 1,432 & $-0,024$ & 1,421 & $-0,013$ \\
\hline $\mathrm{C} 2-\mathrm{C} 3$ & 1,530 & 1,533 & $-0,003$ & 1,541 & $-0,011$ \\
\hline $\mathrm{C} 3-\mathrm{N} 4$ & 1,354 & 1,436 & $-0,082$ & 1,443 & $-0,089$ \\
\hline $\mathrm{N} 4-\mathrm{C} 10$ & 1,416 & 1,442 & $-0,026$ & 1,455 & $-0,039$ \\
\hline $\mathrm{C} 2-\mathrm{O} 11$ & 1,354 & 1,390 & $-0,036$ & 1,379 & $-0,025$ \\
\hline $\mathrm{C} 3-\mathrm{O} 12$ & 1,195 & 1,235 & $-0,040$ & 1,215 & $-0,020$ \\
\hline N4-O13 & 1,369 & 1,339 & 0,030 & 1,457 & 0,088 \\
\hline $\mathrm{C} 7-\mathrm{O} 14$ & 1,359 & 1,379 & $-0,020$ & 1,380 & $-0,021$ \\
\hline C9-O1 & 1,356 & 1,384 & $-0,028$ & 1,380 & $-0,024$ \\
\hline $\mathrm{O} 14-\mathrm{C} 15$ & 1,408 & 1,424 & $-0,016$ & 1,409 & $-0,001$ \\
\hline H-O11 & 0,952 & 0,961 & $-0,009$ & 0,951 & 0,001 \\
\hline $\mathrm{H}-\mathrm{O} 13$ & 0,950 & 0,978 & $-0,028$ & 0,949 & 0,001 \\
\hline Média & - & - & 0,029 & - & 0,028 \\
\hline $\mathrm{O} 1-\mathrm{C} 2-\mathrm{C} 3$ & 107,51 & 111,69 & $-4,18$ & 112,61 & $-5,10$ \\
\hline $\mathrm{O} 1-\mathrm{C} 2-\mathrm{O} 11$ & 110,00 & 102,30 & 7,70 & 98,66 & 11,34 \\
\hline C2-C3-N4 & 112,79 & 115,07 & $-2,28$ & 115,58 & $-2,79$ \\
\hline C2-C3-O12 & 122,33 & 122,25 & 0,08 & 124,23 & $-1,90$ \\
\hline C3-N4-C10 & 121,17 & 114,20 & 6,97 & 115,85 & 5,32 \\
\hline C3-N4-O13 & 117,02 & 113,90 & 3,12 & 114,25 & 2,77 \\
\hline C7-O14-C15 & 115,64 & 116,29 & $-0,65$ & 116,65 & $-1,01$ \\
\hline Média & - & & 3,57 & - & 4,32 \\
\hline
\end{tabular}

${ }^{\mathrm{a} D i s t a ̂ n c i a ~ d e ~ l i g a c ̧ o ̃ e s ~ e m ~ \AA ̊ . ~ a ̂ n g u l o s ~ e m ~ g r a u s . ~}$

Tabela 4. Comparação das cargas eletrostáticas obtidas pelos métodos HF/6-31G*, AM1 e PM3.

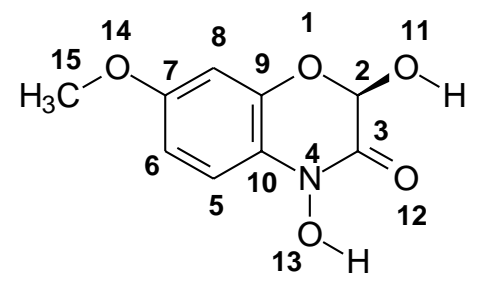

\begin{tabular}{cccccc}
\hline átomo $^{\mathrm{a}}$ & $\mathrm{HF} / 6-31 \mathrm{G}^{*}$ & AM1 & Diferença $^{\mathrm{b}}$ & PM3 & Diferença $^{\mathrm{b}}$ \\
\hline O1 & $-0,408$ & $-0,258$ & $-0,150$ & $-0,274$ & $-0,134$ \\
C2 & 0,613 & 0,061 & 0,552 & 0,150 & 0,463 \\
C3 & 0,512 & 0,792 & $-0,280$ & 0,774 & $-0,262$ \\
N4 & $-0,170$ & $-0,494$ & 0,324 & $-0,373$ & 0,203 \\
C5 & $-0,212$ & 0,012 & $-0,224$ & $-0,045$ & $-0,167$ \\
C6 & $-0,296$ & $-0,365$ & 0,069 & $-0,278$ & $-0,018$ \\
C7 & 0,410 & 0,407 & 0,003 & 0,377 & $-0,033$ \\
C8 & $-0,451$ & $-0,322$ & $-0,129$ & $-0,343$ & $-0,108$ \\
C9 & 0,333 & 0,162 & 0,171 & 0,198 & 0,135 \\
C10 & 0,056 & 0,083 & $-0,027$ & 0,055 & $-0,001$ \\
O11 & $-0,669$ & $-0,458$ & 0,211 & $-0,477$ & $-0,192$ \\
O12 & $-0,557$ & $-0,479$ & 0,078 & $-0,518$ & $-0,039$ \\
O13 & $-0,377$ & $-0,255$ & $-0,122$ & $-0,260$ & $-0,117$ \\
O14 & $-0,443$ & $-0,355$ & $-0,088$ & $-0,381$ & $-0,062$ \\
C15 & 0,187 & 0,036 & 0,151 & 0,271 & - \\
Média & - & - & 0,172 & 0,023 & 0,135 \\
& & 0,047 & & 0,023 & \\
\hline
\end{tabular}

\footnotetext{
${ }^{\mathrm{a} C}$ Cargas calculadas pelo ajuste ao potencial eletrostático; ${ }^{\mathrm{b}} \mathrm{Parâmetro(ab}$ initio) - Parâmetro(semi-empírico)
} 
$\Delta \mathrm{G}_{\mathrm{r}}=-\mathrm{RT} \ln K_{\mathrm{a}}=-2,303 \mathrm{RT} \log K_{\mathrm{a}}$

ou,

$$
\Delta \mathrm{H}_{\mathrm{r}}-\mathrm{T} \Delta \mathrm{S}_{\mathrm{r}}=2,303 \mathrm{RT} \mathrm{p} K_{\mathrm{a}}
$$

Assumindo o equilíbrio de dissociação dos derivados HA de 2 no próton $\mathrm{H}^{+}$e no ânion $\mathrm{A}^{-}$, quer seja pela perda do próton da hidroxila fenólica ou do grupo ácido hidroxâmico, temos que

$\Delta \mathrm{H}_{\mathrm{r}}=\Delta \mathrm{H}_{\mathrm{f}}\left(\mathrm{A}^{-}\right)+\Delta \mathrm{H}_{\mathrm{f}}\left(\mathrm{H}^{+}\right)-\Delta \mathrm{H}_{\mathrm{f}}(\mathrm{HA})$

Substituindo (3) em (2),

$\Delta \mathrm{H}_{\mathrm{f}}\left(\mathrm{A}^{-}\right)+\Delta \mathrm{H}_{\mathrm{f}}\left(\mathrm{H}^{+}\right)-\Delta \mathrm{H}_{\mathrm{f}}(\mathrm{HA})-\mathrm{T} \Delta \mathrm{S}_{\mathrm{r}}=2,303 \mathrm{RT} \mathrm{p} K_{\mathrm{a}}$

ou,

$\Delta \mathrm{H}_{\mathrm{f}}\left(\mathrm{A}^{-}\right)-\Delta \mathrm{H}_{\mathrm{f}}(\mathrm{HA})=2,303 \mathrm{RT} \mathrm{p} K_{\mathrm{a}}+\mathrm{T} \Delta \mathrm{S}_{\mathrm{r}}-\Delta \mathrm{H}_{\mathrm{f}}\left(\mathrm{H}^{+}\right)$

Se o termo $T \Delta S_{\mathrm{r}}$ não for muito diferente para a série de ácidos HA estudados, a equação (5), que representa a diferença entre $\Delta \mathrm{H}_{\mathrm{f}}$ entre o ânion e a forma protonada em função do $\mathrm{p} K_{\mathrm{a}}$ do ácido HA, deve ser uma equação aproximadamente linear. As diferenças de $\Delta \mathrm{H}_{\mathrm{f}}$ calculado entre os ânions hidroxamato e as moléculas neutras dos análogos $\left(\Delta \Delta \mathrm{H}_{\mathrm{f} 1}\right)$ e entre os ânions fenolato e as moléculas neutras dos análogos $\left(\Delta \Delta \mathrm{H}_{\mathrm{f} 2}\right)$, foram comparadas, respectivamente, com os dados de $\mathrm{pK}_{\mathrm{a} 1}$ e $\mathrm{pK}_{\mathrm{a} 2}$, na Tabela 5 .

Os gráficos das Figuras 3 e 4 mostram que o aumento da estabilidade relativa calculada dos ânions em relação às moléculas neutras acompanha o aumento da acidez, com uma correlação linear comparável para os métodos AM1 e PM3, no caso de $\Delta \Delta \mathrm{H}_{\mathrm{f} 1}$ em função de $\mathrm{pK}_{\mathrm{a} 1}$, e ligeiramente superior para o método PM3, no caso de $\Delta \Delta \mathrm{H}_{\mathrm{f} 2}$ em função de $\mathrm{pK}_{\mathrm{a} 2}$. Esta avaliação mostra que os métodos semi-empíricos são capazes de representar o efeito eletrônico relativo de diferentes substituintes sobre uma propriedade química experimental das benzoxazinonas.

\section{METODOLOGIA}

Todas as etapas foram realizadas em computadores Pentium II $333 \mathrm{Mhz}$, sob o sistema operacional Windows98. A construção das matrizes correspondentes às estruturas estudadas e o cálculo no nível $a b$ initio $6-31 \mathrm{G}^{*}$ foram feitos com o programa

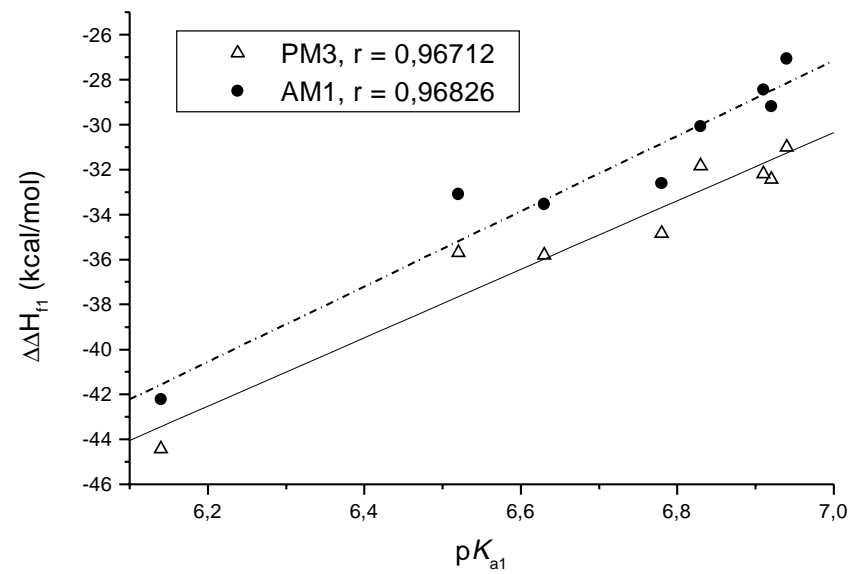

Figura 3. Gráfico de $\Delta \Delta H_{f l}\left[\Delta H_{f f}\right.$ hidroxamato) $-\Delta H_{f(\text { enol })] \text { calculado }}$ pelos métodos PM3 e AM1contra $p K_{a 1}$ para a série de 2,4-diidróxi1,4-benzoxazin-3-onas ${ }^{20}$.

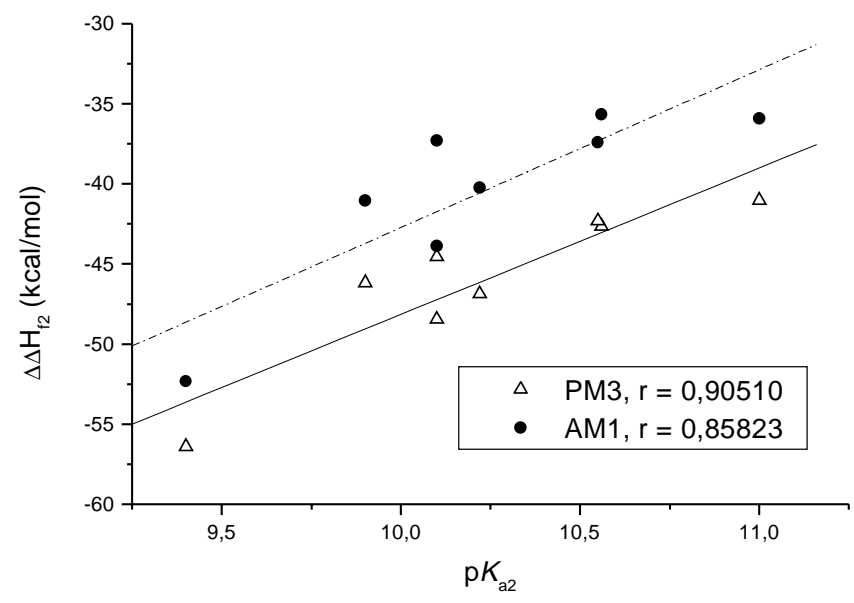

Figura 4. Gráfico de $\Delta \Delta H_{f 2}\left[\Delta H_{f}\right.$ fenolato $)-\Delta H f($ enol)] calculado pelos métodos PM3 e AM1contra $p K_{a 2}$ para a série de 2,4-diidróxi-1,4benzoxazin-3-onas ${ }^{20}$

PC Spartan Plus $1.5^{21}$. As cargas atômicas foram calculadas por ajuste ao potencial eletrostático. A otimização das estruturas dos $\mathrm{Hx}$ com os métodos semi-empíricos AM1 e PM3 foi

Tabela 5. Comparação entre resultados experimentais ${ }^{20}$ de acidez dos grupos ácido hidroxâmico $\left(\mathrm{pK}_{\mathrm{a} 1}\right)$ e fenol $\left(\mathrm{pK}_{\mathrm{a} 2}\right)$ e dados de $\Delta \mathrm{H}_{\mathrm{f}}$ relativo para benzoxazolinonas, calculados pelos métodos AM1 e PM3<smiles>[X]c1ccc(N(O)C(=O)C=O)c(O)c1</smiles>

\begin{tabular}{|c|c|c|c|c|c|c|}
\hline \multirow[t]{2}{*}{$X$} & \multicolumn{2}{|c|}{$\Delta \Delta \mathrm{H}_{\mathrm{f} 1}^{\mathrm{b}}$} & \multirow[t]{2}{*}{$\mathrm{pK}_{\mathrm{a} 1}^{\mathrm{a}}$} & \multicolumn{2}{|c|}{$\Delta \Delta \mathrm{H}_{\mathrm{f} 2}{ }^{\mathrm{c}}$} & \multirow[t]{2}{*}{$\mathrm{pK}_{\mathrm{a} 2}{ }^{\mathrm{a}}$} \\
\hline & AM1 & PM3 & & AM1 & PM3 & \\
\hline $\mathrm{t}-\mathrm{Bu}$ & 6,94 & $-27,061$ & $-30,993$ & 11,0 & $-35,937$ & $-41,026$ \\
\hline $\mathrm{H}$ & 6,91 & $-28,448$ & $-31,847$ & 10,55 & $-37,412$ & $-42,655$ \\
\hline $\mathrm{MeO}$ & 6,92 & $-29,187$ & $-32,195$ & 10,1 & $-37,299$ & $-42,333$ \\
\hline $\mathrm{Me}$ & 6,83 & $-30,068$ & $-32,443$ & 10,56 & $-35,659$ & $-44,554$ \\
\hline $\mathrm{Cl}$ & 6,78 & $-32,614$ & $-34,834$ & 10,22 & $-40,241$ & $-46,867$ \\
\hline $\mathrm{CO}_{2} \mathrm{Me}$ & 6,52 & $-33,084$ & $-35,688$ & 9,90 & $-41,059$ & $-46,169$ \\
\hline $\mathrm{F}$ & 6,63 & $-33,534$ & $-35,815$ & $\sim 10,1$ & $-43,879$ & $-48,455$ \\
\hline $\mathrm{NO}_{2}$ & 6,14 & $-42,211$ & $-44,418$ & 9,40 & $-52,311$ & $-56,408$ \\
\hline
\end{tabular}

${ }^{\mathrm{a}}$ Ref. $20 ;{ }^{\mathrm{b}} \Delta \mathrm{H}_{\mathrm{f}}\left(\right.$ ânions hidroxamato) $-\Delta \mathrm{H}_{\mathrm{f}}$ (moléculas neutras), kcal $/ \mathrm{mol} ;{ }^{\mathrm{c}} \Delta \mathrm{H}_{\mathrm{f}}$ (ânions fenolato) $-\Delta \mathrm{H}_{\mathrm{f}}(\mathrm{moléculas} \mathrm{neutras})$, kcal/mol. 
feita através do programa Mopac $6.0^{22}$ até uma norma de gradiente menor do que $0,1 \mathrm{kcal} /\left(\AA^{\circ}\right.$ ou $\left.{ }^{\circ}\right)$.

\section{CONCLUSÕES}

Com relação à avaliação da estabilidade relativa das conformações dos diastereoisômeros $Z$ e $E$ das formas ácido hidroxâmico e ácido hidroxímico do ácido formoidroxâmico, o método semi-empírico PM3 mostrou um desempenho superior ao método AM1, em comparação com dados do método $a b$ initio MP2/6-31G (d). Ambos os métodos, em comparação com dados do método ab initio $\mathrm{HF} / 6-31 \mathrm{G}^{*}$, produziram previsões razoáveis da estrutura de DIMBOA, mas os dados de cargas eletrostáticas apresentam grandes diferenças para ambos os métodos semi-empíricos, embora o desempenho do método PM3 tenha sido melhor. Foram encontradas boas correlações entre a estabilidade relativa, calculadas pelos métodos AM1 e PM3, dos ânions fenolato e hidroxamato e das formas protonadas de derivados da forma aldólica de DIMBOA, e dados experimentais de $\mathrm{p} K_{\mathrm{a}}$. Esta avaliação mostra que os métodos AM1 e PM3 são capazes de representar o efeito eletrônico relativo de substituintes sobre uma propriedade química experimental das benzoxazinonas. O conjunto dos resultados sugere que os métodos semi-empíricos avaliados podem, com algumas restrições, serem aplicados ao estudo de sistemas que contenham a função ácido hidroxâmico, com alguma vantagem para o método PM3 em relação ao método AM1. Somada à velocidade dos métodos semi-empíricos, esta conclusão aponta para o seu uso em estudos da interação entre enzimas e $\mathrm{Hx}$.

\section{AGRADECIMENTOS}

Os autores agradecem ao programa PIBIC (CNPq/UFRRJ) pela bolsa de Iniciação Científica e à Faperj pelo apoio financeiro.

\section{REFERÊNCIAS}

1. Berenbaun, M.R.; Radovsky, F. J.; Resh, V. H.; Ann. Rev. Entomol. 1998, 43, 1.

2. Niemeyer, H. M.; Phytochemistry 1988, 27, 3349.

3. Niemeyer, H. M.; Pesel, E.; Copaja, S. V.; Bravo, H. R.; Franke, S.; Francke, W.; Phytochemistry 1989, $28,447$.
4. Niemeyer, H. M.; Pesel, E.; Franke, S.; Francke, W.; Phytochemistry 1989, 28, 2307.

5. Gianoli, E.; Niemeyer, H. M.; J. Chem. Ecol. 1997, 23, 2695.

6. Hashimoto, Y.; Shudo, K.; Phytochemistry 1996, 43, 551

7. Copaja, S. V.; Bravo, H. R.; Niemeyer, H. M.; J. Org. Chem. 1986, 51, 3542.

8. Queirolo, C. B.; Andreo, C. S.; Niemeyer, H. M.; Corcuera, L. J.; Phytochemistry 1983, 22, 2455.

9. Yamamoto, M.; Tsujishita, H.; Hori, N., Ohishi, Y.; Inoue, S.; Ikeda, S.; Okada, Y.; J. Med. Chem. 1998, $41,1209$.

10. Cuevas, L; Niemeyer, H. M.; Phytochemistry 1993, 34, 983.

11. Leszczynski, B.; Matok, H; Dixon, A. F. G.; J. Chem Ecol. 1992, 18, 1189.

12. Perez, F. J.; Niemeyer, H. M.; Phytochemistry 1989, $28,1597$.

13. Cuevas, L.; Niemeyer, H. M.; Perez, F. J.; Phytochemistry 1990, 29, 1429.

14. Pople, J. A. e Beveridge, D. L.; Approximate Molecular Orbital Theory; McGraw-Hill, New York, 1970. Segal, J. A. (Ed.); Semi-empirical Methods of Electronic Structure Calculation; Plenum Press, New York, 1977.

15. Clark, T. A.; A Handbook of Computational Chemistry; Wiley, New York, 1985. Hirst, D. M.; A Computational Approach to Chemistry; Blackwell Scientific Publications, Oxford, 1990; p 85.

16. Manunza, B.; Deiana, S.; Pintore, M.,; Solinas, V.; Gessa, C.; J. Mol. Struc. (Theochem) 1997, 419, 33; Wasserman, Z. R.; Hodge, C. N.; Proteins-Struct. Func. Gen. 1996, 24, 227.

17. Dewar, M. J. S.; Zoebisch, E. G.; Healy, E. F.; Stewart, J. J. P.; J. Am. Chem. Soc. 1985, 107, 3902.

18. Stewart, J. J. P.; J. Comput. Chem. 1989, 1, 209.

19. Brink, C. P.; Crumbliss, A. L.; J. Mol. Struc. (Theochem) 1996, 370, 25.

20. Atkinson, J.; Morand, P.; Arnason, J. T.; J. Org. Chem. 1991, 56, 1788.

21. Deppmeier, B. J.; Driessen, A. J.; Hehre, A. J.; Hume, T. T.; Johnson, J. A; Klunzinger, P. E.; Lou, L.; Yu, J.; Wavefunction, Inc., Irvine, EUA.

22. Stewart, J. J. P., QCPE 455, disponível pela Indiana University, Bloomington, EUA. 